\title{
A CROSSED MOLECULAR BEAMS STUDY ON THE FORMATION OF THE EXOTIC CYANOETHYNYL RADICAL IN TITAN'S ATMOSPHERE
}

\author{
X. Gu ${ }^{1}$, R. I. Kaiser ${ }^{1,7}$, A. M. Mebel ${ }^{2}$, V. V. Kislov ${ }^{2}$, S. J. Klippenstein ${ }^{3}$, L. B. Harding ${ }^{3}$, M. C. Liang ${ }^{4,5}$, and Y. L. Yung ${ }^{6}$ \\ ${ }^{1}$ Department of Chemistry, University of Hawaii at Manoa, Honolulu, HI 96822, USA \\ ${ }^{2}$ Department of Chemistry and Biochemistry, Florida International University, Miami, FL 33199, USA \\ ${ }^{3}$ Chemical Sciences and Engineering Division, Argonne National Laboratory, Argonne, IL 60439, USA \\ ${ }^{4}$ Research Center for Environmental Changes, Academia Sinica, Taipei, Taiwan \\ ${ }^{5}$ Graduate Institute of Astronomy, National Central University, Jhongli, Taiwan \\ ${ }^{6}$ Division of Geological and Planetary Sciences, Caltech, Pasadena, CA 91125, USA \\ Received 2009 May 4; accepted 2009 June 3; published 2009 August 6
}

\begin{abstract}
The reaction of the dicarbon molecule $\left(\mathrm{C}_{2}\right)$ in its ${ }^{1} \Sigma_{\mathrm{g}}{ }^{+}$electronic ground state with hydrogen cyanide $\operatorname{HCN}\left(\mathrm{X}^{1} \Sigma^{+}\right)$is investigated in a crossed molecular beam setup to untangle the formation of the cyanoethynyl radical $\mathrm{CCCN}\left(\mathrm{X}^{2} \Sigma^{+}\right)$in hydrocarbon-rich atmospheres of planets and their moons such as Titan. Combined with electronic structure and rate theory calculations, we show that this elementary reaction is rapid, has no entrance barriers, and yields CCCN via successive rearrangements of the initial $\mathrm{HC}_{3} \mathrm{~N}$ collision complex to the cyanoacetylene intermediate $(\mathrm{HCCCN})$ followed by unimolecular decomposition of the latter without exit barrier. New photochemical models imply that this radical could serve as a key building block to form more complex molecules as observed in situ by the Cassini spacecraft, ultimately leading to organic aerosol particles, which make up the orange-brownish haze layers in Titan's atmosphere.
\end{abstract}

Key words: astrochemistry - methods: laboratory - molecular processes - planets and satellites: general scattering

Online-only material: color figures

\section{INTRODUCTION}

Since its discovery by Huygens in 1655, Saturn's moon Titan has been a subject of vigorous research, predominantly due to its role as a "natural laboratory," yielding vital clues—at the frozen stage —on the chemical evolution of proto-Earth (Atreya 2007). Titan's orange haze layers present the most prominent visible features. They play a crucial role in controlling Titan's weather and chemistry (Lorenz et al. 2008), absorb the destructive ultraviolet radiation from the Sun ("prebiotic ozone") to protect latent astrobiologically important molecules in the lower atmosphere and on the surface (Atreya et al. 2006), and are thought to resemble terrestrial aerosol layers before life developed on Earth more than 3.8 billion years ago (Raulin 2008). However, despite the crucial role of Titan's haze layers, the underlying chemical processes which control their formation have been the least understood to date (Vuitton et al. 2006; Hebrard et al. 2007). This mechanism is of paramount importance to comprehend the processing of hydrocarbon-rich atmospheres and to the interpretation of the atmospheric data of current (CassiniHuygens) and future probes to the outer solar system. Current reaction networks simulating Titan's atmosphere suggest that the chemistry is driven predominantly by solar ultraviolet photons (Vuitton et al. 2006; Hebrard et al. 2007). This process generates trace species such as, polyacetylenes and cyanopolyacetylenes, diacetylene $(\mathrm{HCCCCH})$, and cyanoacetylene $(\mathrm{HCCCN})$ being the simplest members of each class. These unsaturated gaseous molecules are thought to "polymerize" to aerosols and so-called tholins, a term coined by Sagan defining a complex mixture of organics observed after irradiating Titan-analogous gas mixtures, which are believed to be present on the surface of Titan and in the atmospheric haze surrounding it. However, contemporary photochemical models suffer from inadequate laboratory data

\footnotetext{
7 Corresponding author email: ralfk@ hawaii.edu
}

with the products and rate constants for many of the reactions being purely speculative. Such shortcomings are reflected in the hitherto poor quantitative agreement between models and the full set of stratospheric molecular mixing ratios inferred from Cassini-Huygens observations (Hebrard et al. 2007). Hence, the mechanism for formation of the haze layers, via the conversion of simple molecules to aerosols, remains uncertain. Since the macroscopic alteration of Titan's atmosphere consists of multiple elementary reactions that are a series of bimolecular encounters between molecules, a detailed understanding of the mechanisms involved at the most fundamental, microscopic level is crucial.

In this paper, we present a crossed molecular beam investigation-combined with electronic structure and rate theory calculations - on the reaction of the important dicarbon molecule $\left(\mathrm{C}_{2} ; \mathrm{X}^{1} \Sigma_{\mathrm{g}}{ }^{+}\right)$with hydrogen cyanide $\left(\mathrm{HCN} ; \mathrm{X}^{1} \Sigma^{+}\right)$ to untangle the synthesis of the cyanoethynyl radical $\left(\mathrm{CCCN}\left(\mathrm{X}^{2} \Sigma^{+}\right)\right)$via a cyanoacetylene intermediate (HCCCN; $\mathrm{X}^{1} \Sigma^{+}$) in the gas phase of Titan's atmosphere (Equation (1)). We also demonstrate via atmospheric modeling that this transient species may present the key building block in the formation of Titan's organic haze. This system represents the prototype reaction of ubiquitous $\mathrm{HCN}$ molecules with the simplest, diatomic transient species, $\mathrm{C}_{2}$, to form $\mathrm{CCCN}$ in hydrocarbon-rich atmospheres of planets and their satellites via a single collision event. The Cassini's Composite Infrared Spectrometer (CIRS) quantified mixing ratios of $\mathrm{HCN}$ to be between $1 \times 10^{-6}$ and $1 \times$ $10^{-7}$ (Flasar et al. 2005) and $7.8 \times 10^{-7}\left(70^{\circ} \mathrm{N}\right)$ and $5.7 \times 10^{-8}$ $70^{\circ} \mathrm{S}$ (Coustenis et al. 2007). At $90^{\circ} \mathrm{S}$ to $20^{\circ} \mathrm{S}$, the $\mathrm{HCN}$ abundance is fairly constant with volume mixing ratios of $1 \times 10^{-7}$ at the $3 \mathrm{mbar}$ level; in northern latitudes, this increases steadily to $4 \times 10^{-7}$ at $60^{\circ} \mathrm{N}$ (Teanby et al. 2006). In Saturn's moon Titan, for instance, photons $(\lambda<200 \mathrm{~nm})$ can penetrate to the stratosphere and mesosphere and dissociate acetylene to atomic hydrogen plus an ethynyl radical $\left(\mathrm{C}_{2} \mathrm{H}\right.$; Jackson et al. 1996). 
The latter can absorb a second photon to yield the $\mathrm{C}_{2}$ species (Becker 2000). This process is similar to the formation of $C_{2}$ from acetylene in comets. The reaction of $\mathrm{C}_{2}$ with omnipresent $\mathrm{HCN}$ is anticipated to form $\mathrm{CCCN}$ in Titan's atmosphere.

$$
\begin{aligned}
\mathrm{C}_{2}\left(\mathrm{X}^{1} \Sigma_{g}^{+}\right) & +\mathrm{HCN}\left(\mathrm{X}^{1} \Sigma^{+}\right) \rightarrow \operatorname{HCCCN}\left(\mathrm{X}^{1} \Sigma^{+}\right) \rightarrow \operatorname{CCCN}\left(\mathrm{X}^{2} \Sigma^{+}\right) \\
& +\mathrm{H}\left({ }^{2} \mathrm{~S}_{1 / 2}\right) .
\end{aligned}
$$

\section{THEORETICAL DETAILS}

Our electronic structure calculations were conducted at a level of theory high enough to predict relative energies of all local minima, transition states, and reaction products to a precision of about $5 \mathrm{~kJ} \mathrm{~mol}^{-1}$. In detail, molecular geometries and vibrational frequencies of reactants, intermediates, and transition states were calculated at the hybrid density functional B3LYP/6-311G** level of theory (Becke 1993) using the Gaussian 98 package (Frisch et al. 1998). Relative energies were refined utilizing the coupled cluster $\operatorname{CCSD}(\mathrm{T})$ method as implemented in the MOLPRO program package (Werner \& Knowles 2003) with extrapolation to the complete basis set (CBS) limit. To achieve this, we computed CCSD(T) total energies for each stationary point with Dunning's correlationconsistent cc-pVDZ, cc-pVTZ, cc-pVQZ, and cc-pV5Z basis sets (Dunning 1989) and projected them to $\operatorname{CCSD}(\mathrm{T}) / \mathrm{CBS}$ total energies (Peterson \& Dunning 1995). Since the addition of singlet $\mathrm{C}_{2}$ to $\mathrm{HCN}$ has no entrance barrier (see below), thermal addition rate constants were computed using variable reaction coordinate transition state theory employing directly determined CASPT2 energies (Klippenstein et al. 2006). The predicted rate constants are expected to be accurate to about $40 \%$ or better.

\section{EXPERIMENTAL DETAILS}

The crossed beam experiments are carried out under single collision conditions coupling a dicarbon, $\mathrm{C}_{2}\left(\mathrm{X}^{1} \Sigma_{\mathrm{g}}{ }^{+}\right)$, source with a universal crossed molecular beam machine (Gu et al. 2006). The $266 \mathrm{~nm}$ output of a Neodymium-yttrium-aluminumgarnet laser is focused onto a rotating carbon rod and ablated species are seeded into neon carrier gas. The supersonic beam of in situ generated dicarbon molecules is chopped and crosses a pulsed $\mathrm{HCN}$ beam $(5 \% \mathrm{HCN}$ seeded in helium) perpendicularly in the interaction region at a collision energy of $22.4 \pm 0.4 \mathrm{~kJ}$ $\mathrm{mol}^{-1}$ and of $25.8 \pm 0.5 \mathrm{~kJ} \mathrm{~mol}^{-1}$. Peak velocities of the $\mathrm{C}_{2}$ beam are $961.3 \pm 22.4$ and $1208.8 \pm 29.7 \mathrm{~ms}^{-1}$ at lower and higher collision energy, respectively, with speed ratios of $5.21 \pm 0.38$ and $3.49 \pm 0.20$ for these segments. The HCN beam segment crossing the $\mathrm{C}_{2}$ pulse is characterized by a peak velocity of $1611.9 \pm 5.7 \mathrm{~ms}^{-1}$ and speed ratio of $15.3 \pm 2.7$. Note that the ablation beam also contains ground-state carbon atoms $\mathrm{C}\left({ }^{3} \mathrm{P}_{\mathrm{j}}\right), \mathrm{C}_{2}$ in their excited triplet state $\left(\mathrm{a}^{3} \Pi\right)$, and tricarbon molecules. However, atomic carbon does not react with $\mathrm{HCN}$ to form $\mathrm{C}_{3} \mathrm{~N}$ molecules (Mebel \& Kaiser 2002). Our calculations show that $\mathrm{C}_{2}\left(\mathrm{a}^{3} \Pi\right)$ can add to the carbon or nitrogen atom of $\mathrm{HCN}$ via barriers of 35.5 and $29.3 \mathrm{~kJ} \mathrm{~mol}^{-1}$, respectively. Since these barriers are above the collision energies in our experiments, triplet $\mathrm{C}_{2}$ reactions with $\mathrm{HCN}$ are neither feasible in our laboratory nor in Titan's atmosphere. Finally, the addition of tricarbon to unsaturated bonds is hindered by entrance barriers larger than our collision energy (Kaiser et al. 2001). Also, we did not see reactive scattering signal at masses higher than $m / z=50$. Therefore, the only species in the primary beam reacting with $\mathrm{HCN}$ are ground-state $\mathrm{C}_{2}$ molecules.
The reactively scattered products were monitored by a quadrupole mass spectrometric detector operated in the timeof-flight (TOF) mode at a constant mass-to-charge ratio $(\mathrm{m} / \mathrm{z})$ after electron-impact ionization of the molecules at $80 \mathrm{eV}$ at an emission current of $2 \mathrm{~mA}$. The detector is rotatable within the plane defined by the $\mathrm{C}_{2}$ and $\mathrm{HCN}$ beams; this allows recording angular resolved TOF spectra and by integrating the TOF spectra at the laboratory angles - the laboratory angular distribution. The latter reports the integrated intensity of an ion of distinct $\mathrm{m} / \mathrm{z}$ versus the laboratory angle. To gain additional information on the chemical dynamics and underlying reaction mechanism, the TOF spectra and laboratory angular distribution were fit and transformed into the center-of-mass (CM) reference frame using a forward-convolution routine ( $\mathrm{Gu}$ et al. 2006). This approach initially presumes the angular flux distribution $\mathrm{T}(\theta)$ and the translational energy flux distribution $\mathrm{P}\left(\mathrm{E}_{\mathrm{T}}\right)$ in the $\mathrm{CM}$ assuming mutual independence. The laboratory data (TOF spectra and the laboratory angular distribution) are then calculated from these $\mathrm{T}(\theta)$ and $\mathrm{P}\left(\mathrm{E}_{\mathrm{T}}\right)$ and convoluted over the apparatus functions to obtain a simulation of the experimental data. The crucial output of this fitting routine is the product flux contour map, $I(\theta, u)=$ $P(u) \times T(\theta)$ which plots the intensity of the reactively scattered products $(I)$ as a function of the CM scattering angle $(\theta)$ and product velocity $(u)$. This plot is called the reactive differential cross section and can be seen as the image of the chemical reaction.

\section{THEORETICAL RESULTS}

The ab initio investigations suggest that on the singlet surface, $\mathrm{C}_{2}$ adds without entrance barrier either to the terminal nitrogen atom or to the carbon-nitrogen triple bond of $\mathrm{HCN}$ forming collision complexes [i0] and [i1], respectively (Figure 1). These intermediates are 243 and $177 \mathrm{~kJ} \mathrm{~mol}^{-1}$ lower in energy than the separated reactants and are connected via a transition state located $208 \mathrm{~kJ} \mathrm{~mol}^{-1}$ above [i0]. Intermediate [i1] can isomerize to the thermodynamically more stable isocyanovinylidene carbene [i2]. The ab initio calculations predict that the latter either undergoes a hydrogen shift to yield isocyanoacetylene [i3] or rearranges via a cyano group flip-over forming the cyanovinylidene intermediate [i4]. Both structures can isomerize to the HCCCN molecule [i5]. This species presents the global minimum of the $\mathrm{C}_{3} \mathrm{NH}$ potential energy surface and is stabilized by $571 \mathrm{~kJ} \mathrm{~mol}^{-1}$ with respect to the separated reactants. With the exception of the linear [i0] and [i5] molecules, which have a ${ }^{1} \Sigma^{+}$ electronic ground state, all singlet intermediates are of $\mathrm{C}_{\mathrm{s}} \mathrm{sym}-$ metry with an ${ }^{1} \mathrm{~A}^{\prime}$ ground state. The linear $\mathrm{CCCN}$ in its ${ }^{2} \Sigma^{+}$electronic ground state is the most stable reaction product $(-11 \mathrm{~kJ}$ $\mathrm{mol}^{-1}$ ) and can be formed without exit barrier via unimolecular decomposition from HCCCN [i5] and/or cyanovinylidenecarbene [i4]. Note that the first excited electronic state $\left(A^{2} \Pi\right)$ is located between 18.3 and $28.7 \mathrm{~kJ} \mathrm{~mol}^{-1}$ above the ground state (Botschwina et al. 1993). An alternate product, the bent $\mathrm{C}_{\mathrm{s}}$ symmetric ${ }^{2} \mathrm{~A}^{\prime}$ isocyanoethynyl radical (CCNC) is $120 \mathrm{~kJ} \mathrm{~mol}^{-1}$ less stable than CCCN. Therefore, it is located well above the energy of the separated reactants. Finally, our computational study suggests the overall rate constant to form ground-state CCCN plus atomic hydrogen ranges from $2.7 \times 10^{-10}$ to $1.5 \times 10^{-10}$ $\mathrm{cm}^{3}$ molecule $\mathrm{s}^{-1} \mathrm{~s}^{-1}$ over Titan's relevant temperature range from $90 \mathrm{~K}$ to $200 \mathrm{~K}$. The calculated thermal rate constants between 90 and $200 \mathrm{~K}$ were fitted by the following expression: $k=1.163 \times 10^{-8} T^{-0.8154} \exp (-9.684 / T)$ in $\mathrm{cm}^{3}$ molecule ${ }^{-1}$ $\mathrm{s}^{-1}$. This value is similar to experimentally derived rate constants of a few $10^{-10} \mathrm{~cm}^{3}$ molecule ${ }^{-1} \mathrm{~s}^{-1}$ for the reaction of $\mathrm{C}_{2}$ 


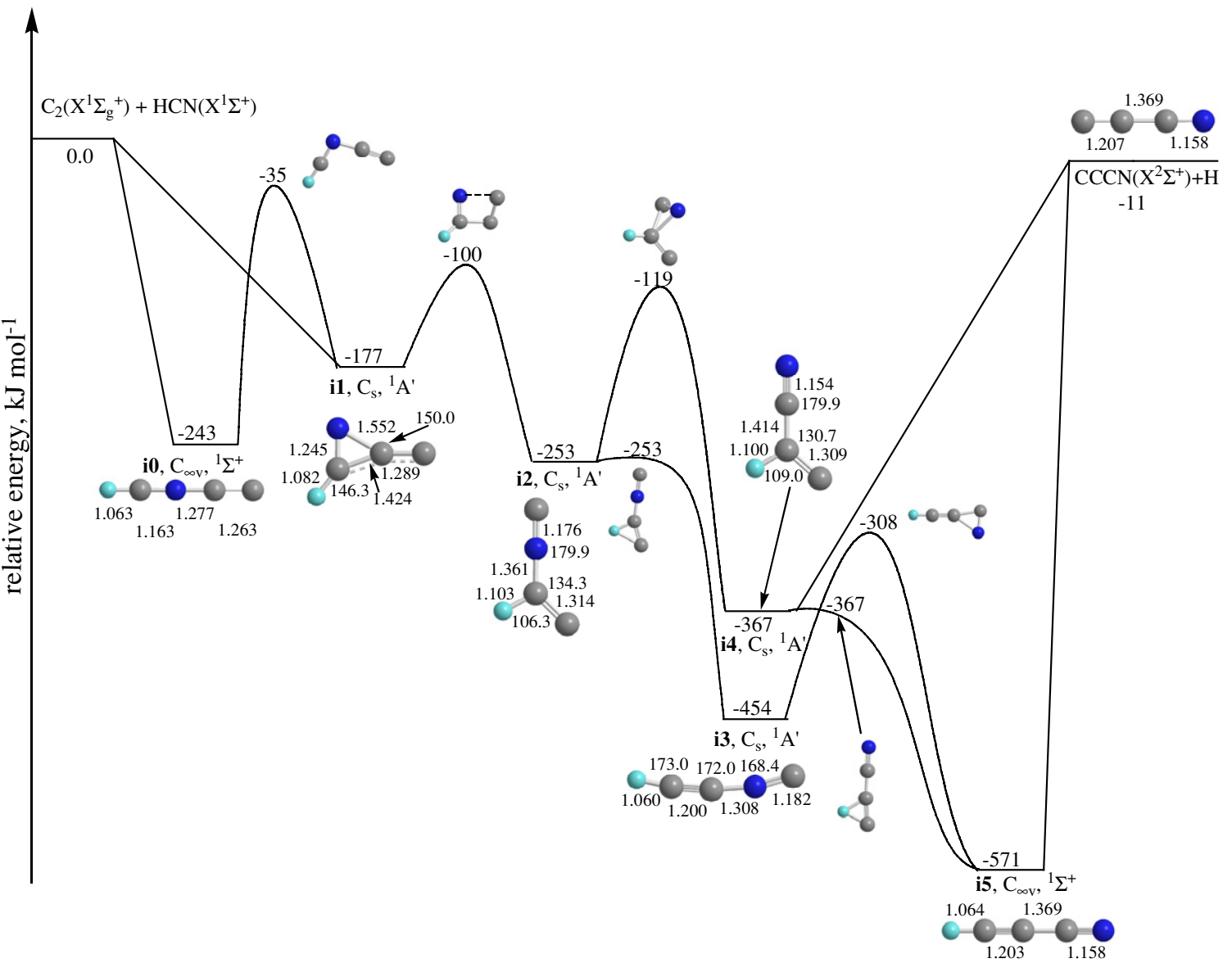

Figure 1. Calculated potential energy surface for the dicarbon, $\mathrm{C}_{2}\left(\mathrm{X}^{1} \Sigma_{g}^{+}\right)$, plus hydrogen cyanide, $\mathrm{HCN}\left(\mathrm{X}^{1} \Sigma^{+}\right)$, reaction to form the linear cyanoethynyl radical, $\mathrm{CCCN}\left(\mathrm{X}^{2} \Sigma^{+}\right)$, plus atomic hydrogen, $\left.\mathrm{H}^{2} \mathrm{~S}_{1 / 2}\right)$. Gray balls depict carbon, dark blue balls nitrogen, and light blue balls hydrogen atoms. Angles are given in degrees and bond lengths in Ångstrom.

(A color version of this figure is available in the online journal.)

with the isoelectronic acetylene $(\mathrm{HCCH})$ reactant (Paramo et al. 2008).

\section{EXPERIMENTAL RESULTS}

TOF spectra are collected at distinct laboratory angles at $m / z=50\left(\mathrm{C}_{3} \mathrm{~N}^{+}\right)$and higher. We observed signal at $m / z=$ $50\left(\mathrm{C}_{3} \mathrm{~N}^{+}\right)$in our experiments (Figures 2 and 3). No products at higher masses were monitored. All TOF spectra could be fit with a single reaction channel accounting for the formation of a molecule of the gross formula $\mathrm{C}_{3} \mathrm{~N}$, which is effectively synthesized via a dicarbon molecule versus hydrogen atom exchange pathway. Since the reaction to form the isocyano radical (CCNC) is strongly endoergic by $110 \mathrm{~kJ} \mathrm{~mol}^{-1}$, this isomer can be ruled out on the basis of our maximum collision energy of only 25.8 $\mathrm{kJ} \mathrm{mol}^{-1}$. Consequently, the cyanoethynyl radical $(\mathrm{CCCN})$ must be the reaction product at $m / z=50$. Note that an alternative hydrogen abstraction channel to form $\mathrm{C}_{2} \mathrm{H}$ plus the cyano radical is calculated to be endoergic by $58 \mathrm{~kJ} \mathrm{~mol}^{-1}$. Therefore, under our experimental conditions and under the low temperatures prevailing in Titan's atmosphere, neither the isocyanoethynyl formation nor the hydrogen abstraction pathway is relevant. These considerations reveal unambiguously that in hydrocarbon-rich planetary environments, the reaction of $\mathrm{C}_{2}$ with $\mathrm{HCN}$ can form CCCN under single collision conditions. This result provides the first experimental identification of a reaction pathway to form
$\mathrm{CCCN}$ in hydrocarbon-rich atmospheres. It should be stressed that, although our collision energies are higher than the relevant conditions in Titan's atmosphere, the formation of cyanoethynyl is, as confirmed by our calculations, the only accessible channel, even down to the very low temperatures of $90-200 \mathrm{~K}$, as prevailing in Titan's atmosphere.

We also investigated the chemical dynamics of the reaction since the structures of the reaction intermediates can influence the underlying reaction mechanism. First, an inspection of the velocity part of the product flux contour maps (Figure 3) and the CM translational energy distributions allows a direct comparison of the theoretically predicted reaction energy with the experimentally derived value. By subtracting the collision energy $\left(E_{C}\right)$ from the maximum translational energy released into the translational degrees of freedom of the reaction products and averaging over both collision energies, the reaction was found to be exoergic by $25 \pm 12 \mathrm{~kJ} \mathrm{~mol}^{-1}$. These data are in good agreement with our ab initio data of $11 \pm 5 \mathrm{~kJ} \mathrm{~mol}^{-1}$. Second, both translational energy distributions peak at zero translational energy. This pattern is a strong indication that the reaction intermediate(s) decompose via a loose exit transition state involving a simple bond rupture process. Thirdly, the flux velocity contour maps show intensity covering the complete scattering range from $\theta=0^{\circ}$ to $\theta=180^{\circ}$. This pattern is indicative of indirect scattering dynamics involving the formation of reaction intermediate(s) (Levine \& Bernstein 1987), here of the generic formula 

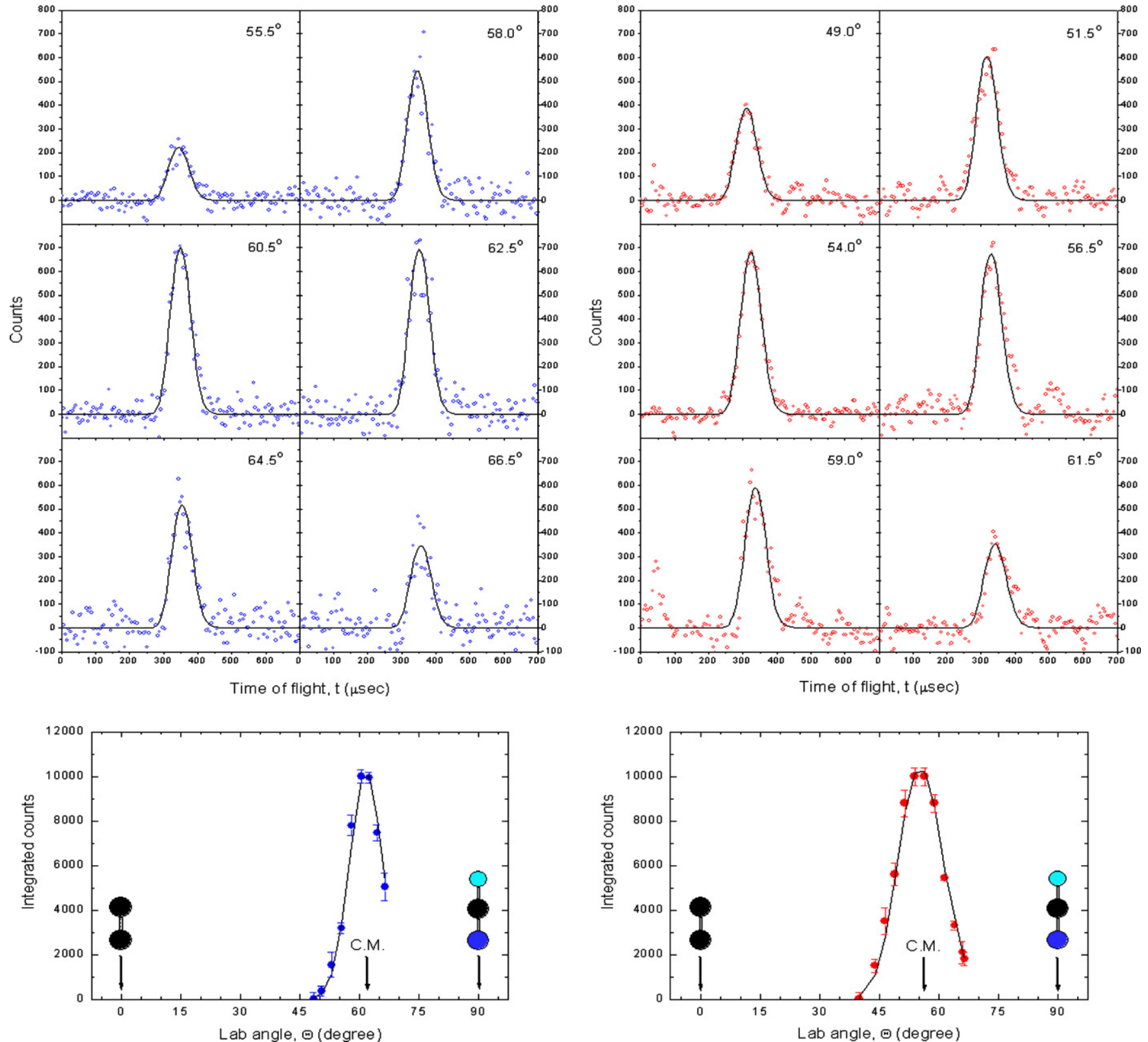

Figure 2. Top: selected time-of-flight (TOF) spectra at mass to charge ratio $m / z=50\left(\mathrm{C}_{3} \mathrm{~N}^{+}\right)$for collision energies of $22.4 \pm 0.4 \mathrm{~kJ}$ mol ${ }^{-1}$ (left) and $25.8 \pm$ $0.5 \mathrm{~kJ} \mathrm{~mol}^{-1}$ (right). Circles denote experimental data, the solid lines the calculated distributions utilizing the best-fit center-of-mass translational and angular distributions (Figure 3). Bottom: corresponding laboratory angular distribution of the reactive scattering signal observed at $m / z=50\left(\mathrm{C}_{3} \mathrm{~N}^{+}\right)$for collision energies of $22.4 \pm$ $0.4 \mathrm{~kJ} \mathrm{~mol}^{-1}$ (left) and $25.8 \pm 0.5 \mathrm{~kJ} \mathrm{~mol}^{-1}$ (right). The filled circles present the experimental data together with the error limits, the solid lines the calculated distribution utilizing the best-fit center-of-mass translational and angular distributions (Figure 3). Black balls depict carbon, dark blue balls nitrogen, and light blue balls hydrogen atoms.

(A color version of this figure is available in the online journal.)

$\mathrm{HC}_{3} \mathrm{~N}$, which decompose(s) to $\mathrm{CCCN}$ via atomic hydrogen loss. As the collision energy increases, the shapes of the flux contour maps and of the CM angular distribution change from a slightly to more forward scattered distribution. This pattern is characteristic of the existence of an osculating $\mathrm{HC}_{3} \mathrm{~N}$ complex (Levine \& Bernstein 1987). With the help of the intensity ratios of the $\mathrm{CM}$ angular distributions at the poles, the reduced mass and relative velocity of the reactants, the moment of inertia of the decomposing HCCCN intermediate $\left(I_{\mathrm{B}}=1.831 \times 10^{-45} \mathrm{~kg}\right.$ $\mathrm{m}^{2}$ ), and the maximum impact parameter leading to reaction $(0.2 \mathrm{~nm})$, we can use the molecular rotation of the linear HCCCN intermediate as a clock and estimate its lifetime to be $4.2 \pm$
$0.6 \mathrm{ps}$ and $0.9 \pm 0.2 \mathrm{ps}$ at lower and higher collision energy, respectively (Miller et al. 1967).

It should be mentioned that the detailed shape of the angular part of the contour map is determined by the disposal of the total angular momentum, the correlation between initial and final angular momentum, and the final rotational angular momentum (Miller et al. 1967). Here, both contour maps are only mildly polarized and depict flux minima at around $90^{\circ}$. This finding strongly indicates that the atomic hydrogen loss of the linear HCCCN intermediate, which is excited to B-like rotations, occurs in the plane of rotation perpendicularly to the total angular momentum vector. Angular momentum 

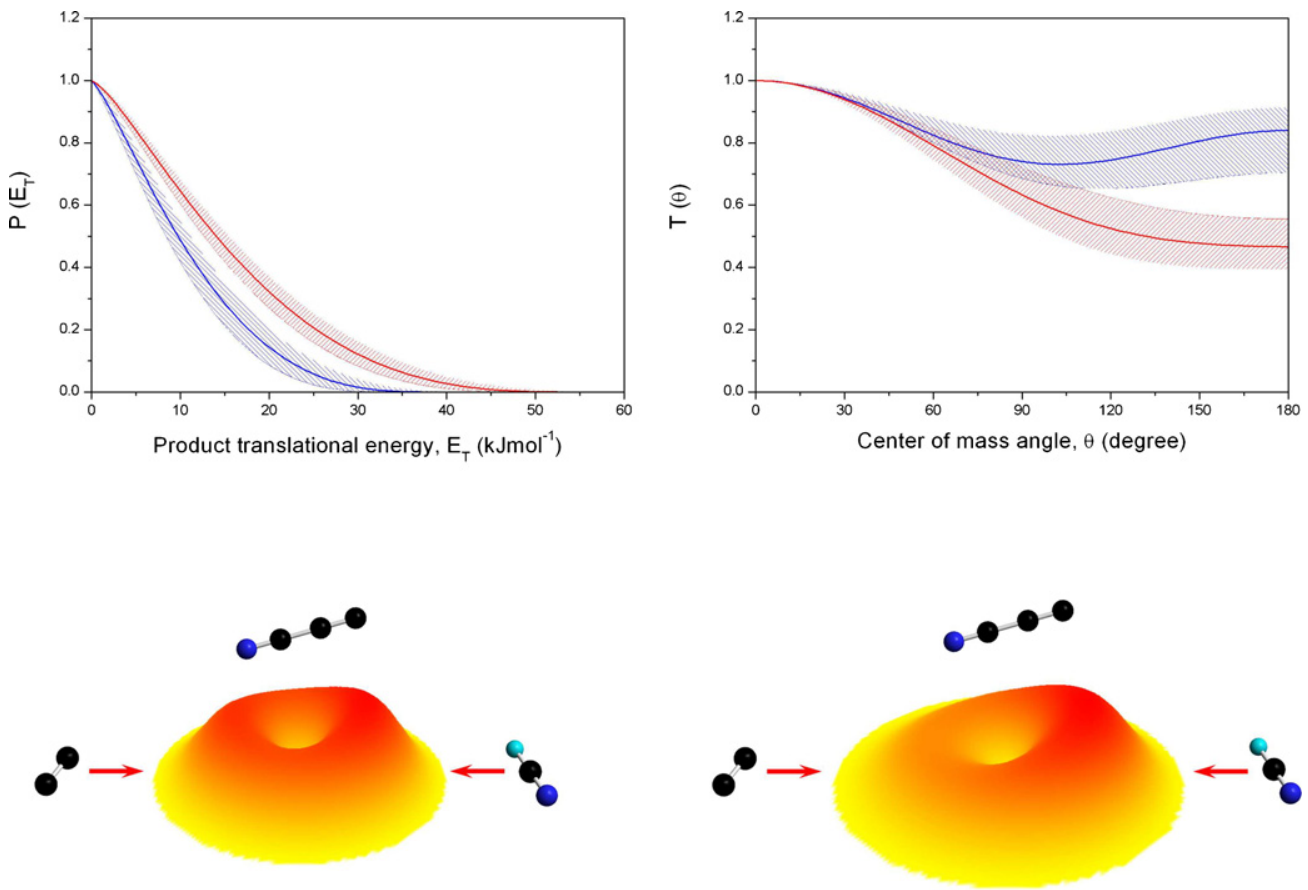

Figure 3. Center-of-mass translational energy distributions (upper left), center-of-mass angular distribution (upper right), and center-of-mass velocity flux contour map (lower left and right at collision energies of $22.4 \pm 0.4 \mathrm{~kJ} \mathrm{~mol}^{-1}$ (blue lines) and $25.8 \pm 0.5 \mathrm{~kJ} \mathrm{~mol}^{-1}$ (red lines), respectively) for the reaction of dicarbon molecules with hydrogen cyanide to form atomic hydrogen plus the cyanoethynyl radical. The shaded areas of the center-of-mass translational energy and angular distributions delimit the range of acceptable fits within the error limits, whereas the solid lines define the 'best fit' functions. The contour lines connect points of constant fluxes (yellow: minimum intensity; red: maximum intensity; the direction of the dicarbon beam is defined as $0^{\circ}$ and of the hydrogen cyanide beam as $180^{\circ}$ ).

(A color version of this figure is available in the online journal.)

conservation also dictates that about $80 \%-90 \%$ of the total angular momentum is channeled onto rotational excitation of the linear $\mathrm{CCCN}$ product. The formation of $\mathrm{CCCN}$ in its ${ }^{2} \Sigma^{+}$electronic ground state also suggests that the hydrogen atom is emitted within the rotation plane of the cyano acetylene molecule since this represents a simple bond rupture of a $\sigma$-symmetric carbonhydrogen bond. On the other hand, the appearance of CCCNs in their $\mathrm{A}^{2} \Pi$ state should likely be reflected in $\mathrm{CM}$ angular distributions holding maxima at or close to $90^{\circ}$ since, in the reverse reaction, the hydrogen atom would add to the $\pi$-symmetric orbital of $\mathrm{CCCN}$ perpendicularly to the rotational plane. Also, based on the energetics of the reaction, the formation of $\operatorname{CCCN}\left(A^{2} \Pi\right)$ is endoergic by at least $7 \mathrm{~kJ} \mathrm{~mol}^{-1}$. Based on the energetics alone, minor amounts of electronically excited CCCNs can be formed in our crossed beam studies, but due to the low temperature, not in Titan's atmosphere. Hence, electronically excited CCCNs formed via unimolecular decomposition of chemically activated HCCCN play no role in the chemical processing of Titan's atmosphere.

\section{ASTROPHYSICAL IMPLICATIOSN AND CONCLUSIONS}

Our combined crossed beam studies and electronic structure calculations provided unprecedented insights into the chemical dynamics on the reaction of $\mathrm{C}_{2}$ with $\mathrm{HCN}$. This reaction proceeds in an indirect fashion via an attack of the electrophilic $\mathrm{C}_{2}$ at either the $\pi$-orbital of the $\mathrm{HCN}$ molecule or the lone pair of the nitrogen. This process proceeds on the ${ }^{2} \mathrm{~A}^{\prime}$ ground state surface, is barrier less, and yields either [i0] or [i1]; our rate constant calculations indicate that the formation of [i0] is favorable. These two isomers readily interconvert, with the latter also isomerizing to the acyclic structure [i2]. Based on the computed barriers, a rearrangement of [i2] to isocyanaoacetylene [i3] and ultimately to HCCCN [i5] is the most favorable isomerization pathway. As verified experimentally and computationally, the latter resides in a deep potential energy well, has a lifetime longer than its rotational period at the lower collision energy, and fragments via a simple carbon-hydrogen bond rupture process through a loose exit transition state to form CCCN. Since the reaction has no entrance barrier, is exoergic, and all transition states involved are lower than the energy of the reactants, this neutral-neutral reaction represents an efficient pathway to produce CCCN in lowtemperature and low-pressure atmospheres of planets and their moons such as Titan. This production of $\mathrm{CCCN}$ will occur via bimolecular reactions in those atmospheric regions where density profiles of photolytically generated $\mathrm{C}_{2}$ and $\mathrm{HCN}$ molecules overlap. Collision times between the $\mathrm{HCCCN}$ intermediate with a nitrogen bath molecule at a temperature range of 100-200 K and relevant pressures of 1 to $1 \times 10^{-6}$ mbar are calculated to be $40-70 \mathrm{~ns}(1 \mathrm{mbar})$ and $40-70 \mathrm{~ms}\left(1 \times 10^{-6} \mathrm{mbar}\right)$. We also derived the lifetimes of the decomposing $\mathrm{HCCCN}$ complexes computationally to be 10 to $17 \mathrm{~ns}$ for the temperature range of 90-200 K of relevance in Titan's atmospheres. This timescale is shorter than the time between collisions required to stabilize this reaction intermediate. Therefore, under the relevant pressure and temperature conditions, only bimolecular reactions are important, and the reaction intermediate cannot be stabilized by a third-body collision.

However, "real" atmospheres present a tricky problem since the chemical evolution of each environment on a "planetary" scale involves multiple reactions that are often intertwined. The 


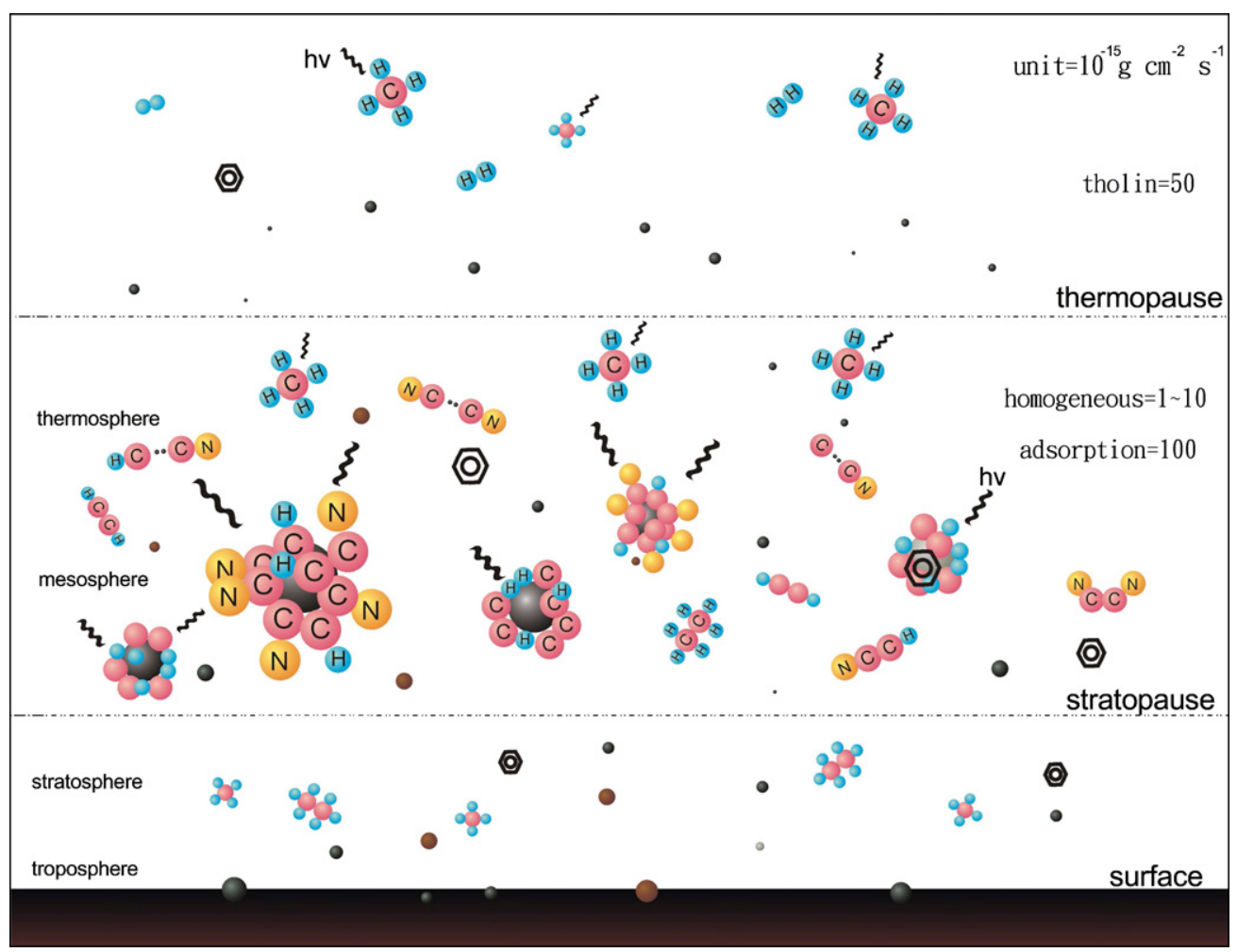

Figure 4. Schematic diagram (not to scale) of gas-phase chemistry and aerosol formation processes in the atmosphere of Titan. Three major sources of aerosols are proposed: ion-neutral chemistry above the thermopause, homogeneous nucleation in the mesosphere and thermosphere, and physicochemical adsorption in the mesosphere and thermosphere. The aerosol production rate via ion-neutral processes is about $5 \times 10^{-14} \mathrm{~g} \mathrm{~cm}^{-2} \mathrm{~s}^{-1}$. The homogeneous nucleation rate from condensable species is $1-10 \times 10^{-15} \mathrm{~g} \mathrm{~cm}^{-2} \mathrm{~s}^{-1}$. The physicochemical processes incorporating the cyanoethynyl radical and its neutral-neutral reactions can contribute as much as $10^{-13} \mathrm{~g} \mathrm{~cm}^{-2} \mathrm{~s}^{-1}$.

(A color version of this figure is available in the online journal.)

complexity of these reactions makes it difficult to investigate the influence of $\mathrm{CCCN}$ on the formation of aerosols and the organic haze layers based on our laboratory and computational studies alone. Therefore, we implemented the reaction of $\mathrm{C}_{2}$ with $\mathrm{HCN}$ into a one-dimensional photochemical model developed for the atmosphere of Titan to realistically link the microscopic scale (chemical reactions under single collision conditions) with the macroscopic setting (Titan's atmosphere; Liang et al. 2007). This newly updated model identified two mechanisms to form CCCN in almost equal amounts: photolysis of HCCCN (HCCCN) and of dicyanoacetylene (NCCCCN) and the neutral-neutral reaction of $\mathrm{C}_{2}$ with $\mathrm{HCN}$ as reported here. Quantitatively spoken, the production and loss rates of CCCN and of $\mathrm{C}_{2}$ are calculated to be $8.7 \times 10^{7}$ molecules $\mathrm{cm}^{-2} \mathrm{~s}^{-1}$ and $1.6 \times 10^{8}$ molecules $\mathrm{cm}^{-2} \mathrm{~s}^{-1}$, respectively. The total loss rate of $\mathrm{HCN}$ is about $8 \times 10^{8}$ molecules $\mathrm{cm}^{-2} \mathrm{~s}^{-1}$, an order of magnitude higher than $\mathrm{HCN}$ lost in the reaction with $\mathrm{C}_{2}$. Therefore, the reaction to form cyanoethynyl has only a minor effect on the $\mathrm{HCN}$ concentration. It is important to indicate that the lifetimes of $\mathrm{C}_{2}$ and of $\mathrm{CCCNs}$ are shorter than the transport time constant; consequently, in those atmospheric regions not exposed to ultraviolet photons, the concentrations of CCCN would be insignificant. The concentrations of long-lived species such as complex cyanopolyacetylenes in polar night regions would be dominantly determined by transport and photochemistry on the day side. Most important, consecutive reactions of $\mathrm{CCCN}$ with acetylene were found to produce significant amounts of cyanodiacetylene $\left(\mathrm{HCCCCCN} ; \mathrm{HC}_{5} \mathrm{~N}\right)$ molecules. Here, the production rate of $2 \times 10^{8}$ molecules $\mathrm{cm}^{-2} \mathrm{~s}^{-1}$ is enhanced by more than $300 \%$ in those models which include the $\mathrm{C}_{2}$ plus $\mathrm{HCN}$ reaction. Consequently, the bimolecular gas phase reaction of $\mathrm{HCN}$ with $\mathrm{C}_{2}$ provides an efficient way for converting atomic nitrogen (its overall production rate is about $10^{9}$ atoms $\mathrm{cm}^{-2} \mathrm{~s}^{-1}$ ) to complex nitrogen compounds. In the atmosphere of Titan, higher order hydrocarbons are greatly enhanced compared with that of giant planets. This is because of the low abundance of hydrogen that prevents the recycling processes to methane. The same mechanism also applies to cyanopolyynes. As the nature of the chemical processes of cyanopolyynes and dicyanopolyynes prefer higher order compounds, which are potentially condensable, the resulting aerosol formation rate from these condensable compounds could be as high as $2 \times 10^{8}$ molecules $\mathrm{cm}^{-2} \mathrm{~s}^{-1}$, a value that is more than $1000 \%$ higher than that previously estimated on the basis of the condensation rate of dicyanodiacetylene alone (NCCCC$\left.\mathrm{CCN} ; \mathrm{C}_{6} \mathrm{~N}_{2}\right)$ - the only species suggested to be condensable in the upper atmosphere of Titan. Figure 4 summarizes our findings. Here, a quantification of the aerosol production rates suggests that neutral-neutral reactions in the mesosphere and thermosphere, including the key reaction of $\mathrm{C}_{2}$ with $\mathrm{HCN}$ and successive reactions of the $\mathrm{CCCN}$ product with hydrocarbons, could be the central mediators of aerosol formation in Titan's atmosphere. Our models suggest that compared to ion-molecule reactions, which dominate the aerosol formation above the thermopause, neutral-neutral reactions can lead to a significantly enhanced aerosol production rate in the lower atmospheres by up to $2000 \%$. Although the absolute condensation rate is one to two orders of magnitude lower than the aerosol production rates derived from the Cassini UVIS measurements (Liang et al. 2007), the condensed species formed provide successive 
condensation centers for subsequent heterogeneous nucleation (Hunten 2006).

\section{SUMMARY}

To summarize, our results are an important step toward a better understanding of the complex, photochemically driven processing of Titan's atmosphere. We have elucidated the formation mechanisms of $\mathrm{CCCN}$ and investigated its role in the formation of aerosols via neutral-neutral reactions in Titan's mesosphere and thermosphere. This investigation leads to the conclusion that $\mathrm{CCCN}$ and its successive reactions may present the dominating production routes of aerosols in Titan's atmosphere. We have also shown that an understanding of the chemical evolution of hydrocarbon-rich atmospheres and aerosol layers must be based on an exact knowledge of detailed elementary reactions in combination with photochemical models, as presented here. We hope that this comprehensive study involving experiments under single collision conditions, electronic structure and rate theory calculations, and global chemical modeling presents a role model that triggers future investigations of key systems relevant to the origin and chemical evolution of hydrocarbon rich atmospheres of planets and their moons.

This work was supported by the US National Science Foundation "Collaborative Research in Chemistry Program" (NSF-CRC; CHE-0627854) and by the National Aeronautics and Space Administration (08-PATM08-0053 to SJK and LBH). We thank Chris Parkinson (University of Michigan) and Hiroshi Imanaka (University of Arizona) for valuable discussions and comments on this manuscript. S.J.K. and L.B.H. acknowledge support by the US Department of Energy, Office of the Basic Energy Sciences, Division of Chemical Sciences, Geosciences, and Biosciences under Contract No. DE-AC02-06CH11357. Y.L.Y. was supported by NASA grant
NNG06GF33G to the California Institute of Technology; M.C.L. was supported in part by NSC grant 97-2628-M-001001 to Academia Sinica.

\section{REFERENCES}

Atreya, S. 2007, Science, 316, 843

Atreya, S. K., et al. 2006, Planet. Space Sci, 54, 1177

Becke, A. D. 1993, J. Chem. Phys., 98, 5648

Becker, K. H., et al. 2000, Z. Phys. Chem., 214, 503

Botschwina, P., Horn, M., Flügge, J., \& Seeger, S. 1993, J. Chem. Soc. Faraday Trans., 89, 2219

Coustenis, A., et al. 2007, Icarus, 189, 35

Dunning, T. H. 1989, J. Chem. Phys., 90, 1007

Flasar, F. M., et al. 2005, Science, 308, 975

Frisch, M. J., et al. 1998, GAUSSIAN 98, Revision A.9 (Pittsburgh, PA: Gaussian, Inc.)

Gu, X., Guo, Y., Zhang, F., Mebel, A. M., \& Kaiser, R. I. 2006, Faraday Discuss., 133,245

Hebrard, E., Dobrijevic, M., Benilan, Y., \& Raulin, F. 2007, Planet. Space Sci., 55,1470

Hunten, D. M. 2006, Nature, 443, 669

Jackson, W. M., et al. 1996, Astrophys. Space Sci., 236, 29

Kaiser, R. I., et al. 2001, Faraday Discuss., 119, 51

Klippenstein, S. J., Georgievskii, Y., \& Harding, L. B. 2006, Phys. Chem. Chem. Phys., 8, 1133

Levine, R. D., \& Bernstein, R. B. 1987, Molecular Reaction Dynamics and Chemical Reactivity (Oxford: Oxford Univ. Press)

Liang, M. C., Yung, Y. L., \& Shemansky, D. E. 2007, ApJ, 661, L199

Lorenz, R. D., et al. 2008, Science, 319, 1649

Mebel, A. M., \& Kaiser, R. I. 2002, ApJ, 564, 787

Miller, W. B., Safron, S. A., \& Herschbach, D. R. 1967, Discuss. Faraday. Soc., 44,108

Paramo, A., Canosa, A., Le Picard, S. D., \& Sims, I. R. 2008, J. Phys. Chem. A, 112, 9591

Peterson, K. A., \& Dunning, T. H. 1995, J. Phys. Chem., 99, 3898

Raulin, F. 2008, Space Sci. Rev., 135, 37

Teanby, N. A., et al. 2006, Icarus, 181, 243

Vuitton, V., Doussin, J.-F., Benilan, Y., Raulin, F., \& Gazeau, M.-C. 2006, Icarus, 185,287

Werner, H. J., \& Knowles, P. J. 2003, MOLPRO, Version 2002.6 (Birmingham: Univ. of Birmingham) 